\title{
Defining and differentiating moral injury's key features
}

\author{
David William Alexander* \\ Centre for Trauma, Asylum \& Refugees, University of Essex, Colchester, UK
}

\begin{abstract}
Although great efforts have been made in recent decades to bring attention to the moral injury discourse as a complementary paradigm to psychiatry in understanding and treating severe forms of combat veteran disorientation, and although a number of treatments for moral injury how now been developed and are under assessment, the discourse is still often ignored, underappreciated, and misunderstood by many professional caregivers. The author contends that this underappreciation can be partly attributed to a poorly-developed internal nosology. This article offers a suggestion for strengthening the development of key, differentiated features within the moral injury discourse, beginning with the author's own experience and categorization of unusual veteran features which cannot be cleanly categorized within the existing spectra of the Diagnostic and Statistical Manual of Mental Disorders.
\end{abstract}

In recent decades, veterans of intense combat operations who develop any kind of painful or disorienting psychological symptoms are increasingly referred to a mental health agency or professional for care, after which they are increasingly placed into summary mental health programs or introduced to packaged treatment plans which address their responses as mental illness, determined by criteria from the traumatic stress disorders spectrum of the psychiatric paradigm. Although it is certain that many combat veterans survivors of adversity do develop Post-Traumatic Stress Disorder (PTSD), the literature suggests that the total number of returning veterans who are candidates for clear PTSD diagnosis totals no more than $20 \%$. Of the other $80 \%$, many still endure considerable distress, even though this distress does not fit precisely into existing psychiatric categories.

At least two dangers therefore arise in contemporary veteran care, given the intense societal focus on PTSD and the limited therapeutic resources available to those veterans whose suffering eludes the diagnostic criteria for PTSD: (a) the pathologization of subclinical or paraclinical veteran distress, in which painful but perhaps ordinary responses to combat-related adversities are treated improperly as "disordered" so that such veterans can access scarce resources, and (b) the medicalization of certain unusual features of distress, which may exist concurrently with PTSD symptoms but which cannot be accounted for in their totality, and which become pressed into psychiatric categories anyway. These dangers have not gone unnoticed in the field. Indeed, the latter danger has gained significant notoriety in American society in conjunction with a steady expansion in both research and practice around the philosophical-therapeutic category of "moral injury," first pioneered by Jonathan Shay [1]. Shay's development of the "moral injury" paradigm to challenge what he considered to be improper and inadequate employment of psychiatric categories to treat his own generation of disoriented combat veterans has provided a continuing platform for the rise of a surprising coalition of complementary theorists from the disciplines of psychology, sociology, medicine, philosophy, world literature, classics, political science and theology.

According to the available literature these theorists have published, however, the moral injury discourse has - for all of its successes - struggled to develop a comprehensive and crisply distinct list of disorienting or distressing features to function nosologically within its own paradigm. The moral injury discourse has gained traction in arguing that veterans who have either experienced a betrayal by leadership or who have enacted, been subject to, or witnessed a violation of what they deeply believe to be right, good, and ultimately meaningful may later develop unusual and enduring forms of shame, meaninglessness, guilt, and grief. The moral injury discourse has probably been unsuccessful, however, in developing clear categories for these unusual forms, and in differentiating them from similar psychiatric criteria related to disorders in various spectra of the Diagnostic and Statistical Manual of Mental Disorders.

As a result, it is clear upon an even cursory review of the literature that although moral injury is purported to be a separate syndrome which should be understood distinctly from PTSD - and, according to dominant themes in the discourse, cannot even be adequately approached using the psychiatric paradigm alone - it is precisely the psychiatric paradigm that is most often used to approach veterans suspected of suffering from moral injury. This may not surprise contemporary American readers, given the strong and speedy emergence of psychiatry in the last 65 years as the primary societal means to understanding and responding to almost all forms of psychological, emotional, and existential distress in the Global West.

There is no doubt that the psychiatric paradigm is an extremely valid and helpful tool when approaching human disorientation in general. However, it seems equally true that if the moral injury paradigm will ever emerge as a separate syndrome which is fully recognized on its own terms, apart from the categories of PTSD in particular and the perhaps bio-physiologically-reductive presuppositions of the psychiatric paradigm in general, more work must be done in terms of nosology and paradigmatic differentiation.

${ }^{\star}$ Correspondence to: David William Alexander, Visiting Fellow, Centre for Trauma, Asylum \& Refugees, University of Essex, Colchester, UK, Tel: 215-9150719; E-mail: dalexaa@essex.ac.uk

Key words: moral injury, veteran, PTSD, treatment, nosology, trauma

Received: September 19, 2018; Accepted: September 26, 2018; Published: September 28, 2018 
This article will attempt a modest offering in that direction. From my own work with more than 25 combat veterans over the past 10 years whose distress is severe enough to impair functioning, has proven resistant to conventional psychotherapeutic treatment methods for PTSD, and contains a strong moral component, I have developed an interest in three complex conditions of distress which I believe should be considered primary features of moral injury: (1) a sense of inner deadening, or diminished connection between consciousness, emotion, and bodily sensation, (2) a persistent, socially-debilitating mistrust, which extends into an eroding capacity for meaning-attribution, and (3) episodes of lustful, exhilarating rage which resist prediction, instate constraint, and external intervention [2]. I will attempt to examine each of these three features below, using the lens of the psychiatric paradigm and exploring whether they may be adequately understood by that paradigm in all of their complexity, uniqueness, and totality, or whether indeed they demand supplementary or even alternative epistemological approaches such as might be more possible after formal enfranchisement within the moral injury paradigm. ${ }^{1}$

\section{Inner deadening}

The feature described above as "inner deadening" after combat features a diminishment of emotional experience and felt embodiment which some veterans conceptualize as a degradation of either the "embodiment of the soul" or the "ensoulment of the body." In this condition veterans develop a sense of extreme emptiness or ghostlikeness, leaving them unable to connect with other people in meaningful ways and unable to access the inner vitality that makes life seem interesting, beautiful, and worth living. This loss of feeling alive, combined with a sense of not being whole, leaves them without a felt connection to themselves, the world, and the circumstances of their lives. They feel haunted, feel distant from the center of themselves, and often desperately long to become whole again. Their experiences seem to describe an intense experience of alienation of which they are acutely aware in the present, and which causes them not only great suffering, but a sense of the painful dissolution of their personalities. This condition seems most likely activated by one or more of the following (a) a betrayal by a leader or close comrade in combat, (b) the loss of one or more leaders or comrades to whom they were very close, or (c) an advanced dehumanization of the enemy. It is completed and will endure if a veteran commits an act that violates his own conscience in the midst of such circumstances. ${ }^{2}$ This description is, I believe, consistent with a number of vignettes included in other works related to moral injury in the existing literature $[1,3,4]$.

This experience of inner deadening seems best accounted for in the psychiatric paradigm by the concept of dissociative depersonalization. In the latest edition of the DSM, this term serves as both a working diagnostic label for a severe accompanying tendency of PTSD and also as the cardinal symptom of a stand-alone dissociative disorder. ${ }^{3}$ Depersonalization Disorder is one of five types of dissociative disorders listed in the DSM-5, and is described as a detachment or estrangement from oneself, coupled with an awareness of this detachment, which cannot be attributable to substance abuse or the result of a general

${ }^{1}$ This phrase "complexity, uniqueness, and totality" is borrowed from Professor Renos Papadopoulos; see Papadopoulos RK, Therapeutic care for refugees: no place like home, Routledge.

${ }^{2}$ I use this concept of activation and completion in the spirit of Freud's nachtraglichkeit. See Kuntstlicher R, "Nachtraglichkeit: the intermediary of an unassimilated impression and experience," Scandinavian psychoanalytical review 17: 101-118.

${ }^{3}$ All references and quotations from "the most recent edition of the DSM" in this chapter will be taken from the first printing (2013) [8] of the American Psychiatric Association's Diagnostic and statistical manual of mental disorders, $5^{\text {th }}$ Edition. medical condition such as the aftermath of a seizure. Although it is classified as a freestanding disorder in its own right, it may, according to the DSM-5, occasionally appear as a feature of PTSD important enough to warrant classification as a sub-type of PTSD. The DSM-5, first published in 2013, was the first edition of the American psychiatric manual to attempt to make any direct clinical connection between depersonalization and PTSD.

Bessel van der Kolk describes dissociative depersonalization as "the outward manifestation of the biological freeze reaction," which for most survivors of devastating events is activated mainly when dissociated material is provoked, but which more rarely can develop into a persistent sense of detachment from oneself [5]. Neziroglu et al. [6] note that, in addition to a sense of detachment from oneself, some adversity survivors who receive this diagnosis describe a sensation of being "encapsulated in a bubble," unable to experience their external environments in some or many ways, or "on autopilot" in some or all of their daily activities. They stress that although this is a rare phenomenon, it is well-documented, and exists in clear distinction from patterned hallucinations or tendencies towards dissociative fragmentations of identity after exposure to devastating events. ${ }^{4}$ People assigned this diagnostic label must be fully aware of not feeling what they used to feel or would normally expect to feel under similar circumstances. Marlene Steinberg notes that people who have been diagnosed with dissociative depersonalization notice a particular loss in their ability to access and deregulate emotion, which in her opinion can contribute to "feeling that the self is strange or unreal, or feeling physically separated from one's body, including out-of-body-experiences... or observing oneself from a distance" $[7,8]$. In her work, Steinberg includes a number of first-hand accounts with clients who describe themselves as feeling like zombies or ghosts, which seem clearly connected to the experiences of a number of veterans whose experiences are recorded in their own language in the literature.

Van der Kolk, Neziroglu, Donnelly, and Steinberg are among those authors in the contemporary psychiatric literature whose descriptions of dissociative depersonalization most strikingly resembles the feature of "inner deadening" as I described it, above. However, in judging whether dissociative depersonalization - as a psychiatric construct can fully and adequately account for the experiences of veterans who have endured this phenomenon, it is important to notice that it is not a popular diagnosis, and that both its theoretical origins and its connection with other psychiatric disorders is not clear. ${ }^{5}$ In fact, the phenomenon of depersonalization seems to have an only tentative connection with dissociation as a larger diagnostic category. R. B. Ulman, who writes from a psychoanalytic perspective, makes the case that the contemporary concept of dissociative depersonalization seems to have directly descended from the Freudian corpus, since Freud first noticed and labeled as depersonalization the phenomenon in which usually under extreme emotional pressure - a person experiences a split in the ego, and afterwards begins to sense self-consciously that "either a piece of reality or a piece of his own self is strange to him" [9]. However, according to Ulman, Freud himself did not pair the terms dissociation and depersonalization to any significant extent, and in fact the concepts have not been clearly linked in the psychiatric literature until very recently. This can perhaps be attributed to the fact that they are not easily compatible, historically speaking. Locating the terms at their points of origin in the psychoanalytic texts, dissociated material is not

${ }^{4}$ Ibid. For this reason, reality testing must be intact for this diagnosis to be assigned.

${ }^{5}$ For an excellent historical argument of this claim, see Dorahy, M. J. \& ven der Hart, O. (2007), Relationship between trauma and dissociation: a historical analysis, Traumatic dissociation: neurobiology and treatment. 
immediately available to the conscious self, whereas depersonalization is something that must be sensed by the conscious self to be known at all.

Dorahy and Hart notice that "the original understanding of dissociation relates to divisions in the personality or consciousness," evincing a complete break between two or more "selves" which are not, in general, able to each be conscious of the other, and each of which contain their own psychobiological systems...collection of memories, affective experiences, behavioral repertoires, and sense of self” [10]. With some alarm, that they note that the concept of dissociation has been widely and perhaps recklessly expanded in contemporary usage, now being used to "account for many and various clinical and... psychological phenomena," some of which do not include a complete break in conscious experience at all. Depersonalization would certainly fit into that latter group. What can account for such a speedy expansion of this diagnostic paradigm, whose original epistemological boundaries have now been seemingly overrun?

Perhaps the ever-evolving pressures of societal dependence on the DSM and its categories provide some account, since such pressures often influence theorists to abandon complexity in order to quickly address phenomena that have not been fully understood. Depersonalization, although rare, is now common enough to warrant inclusion in the DSM, and its proper enfranchisement is not an easy project. In the international community, depersonalization has not been considered a type of dissociative disorder at all; in the ICD-10 it is instead classified as a "not otherwise specified neurotic disorder." American military medical handbooks published as recently as the year 2000 link depersonalization not with dissociation but with extreme emotional exhaustion and moral confusion [11]. As contemporary empirical theorists such as Deihl and Goldberg have noticed, "depersonalization hasn't been studied widely, and its exact cause is still unknown" [12]. It could be easily added that all of its features are not yet known or fully understood, and that its categorization within the dissociative disorders spectrum of the DSM-5 is not necessarily optimal. The DSM-5, then, does recognize the existence of something similar to the phenomenon that I describe as "inner deadening," but its ability to provide a coherent account for the complexity and totality of this phenomenon seems significantly limited. In any case, at no point in the contemporary psychiatric literature is the concept or category of depersonalization connected to a violation of moral beliefs.

\section{Extreme, socially-debilitating mistrust}

The condition described above as "extreme, socially-debilitating mistrust" after combat features the gradual dissolution of a veteran's entire sense of social understanding and public safety after experiences in combat. Such veterans often feel a deep sense of either leadership betrayal or self-horror connected to self-betrayal during and after the war. In the former possibility, a veteran's loss of trust in combat leadership seems to expand into a profound skepticism about the lessons he or she had received throughout life by people in positions of power and authority - and even faith in God or absolute ideals of goodness and truth. Such a veteran may even begin to adopt and incorporate the believe that life has no possibility of a coherent structure, and that there is no reliable way of knowing anything except by personal experience, because nothing is what it seems.

In the latter possibility, a veteran may attempt to isolate him or herself in order to keep loved ones away, perhaps to avoid the potential

${ }^{6}$ See page 13 of the classification of mental and behavioral health disorders in the World Health Organization's (1993) [13] International statistical classification of diseases and related health problems, $10^{\text {th }}$ edition. for "contaminating" loved with the monstrousness they now believe resides within them. Some veterans even describe having absorbed evil in combat, and this is often related to memories of enjoying the act of killing or the commission of inhumane acts. Some seeminglyconnected aspects of this phenomenon include impulses towards homicide and suicide, painful identification with monstrous images or memories, and a sense of having been overtaken by evil and being no longer in control. Both the former and latter possibilities are, I believe, consistent with a number of vignettes included in other works related to moral injury in the existing literature [13-19].

In beginning to examine this painful and unusual feature through the lens of the psychiatric paradigm, it may first be important to notice a few facts about the American Psychiatric Association's (APA's) Diagnostic and Statistical Manual of Mental Disorders (DSM) [20,21]. The APA has made more significant changes to its classification of trauma-related disorders in the last four decades than it has made with any other spectrum of disorders. This perhaps belies the growing importance of the concept of trauma in American social consciousness, and on the corresponding social pressures for American professionals to develop an adequate understanding of trauma and its treatment. Such growth in importance would not have been easy to predict at the time of the initial inclusion of PTSD into the DSM, in 1980. "War neurosis" had been eliminated from the psychiatric nomenclature in the decades preceding the Vietnam War, due to its reputation as an imprecise and increasingly obsolete category. At the time that the DSM-III was being finalized, in the late 1970s, the members of the subcommittee on anxiety disorders - like most Americans - were becoming aware of the severe disorientation of a large number of returning Vietnam veterans, but were unsure of whether or not to recreate a category of combat-related distress. Eventually, they decided to accept influence from both American military psychiatry and Department of Veterans Affairs (VA) psychiatry in developing such a category, and to assign the disorientation of these veterans a label: post-traumatic stress disorder. The newly minted disorder would be associated with three cardinal symptoms, which would serve as its diagnostic criteria: recurrent and intrusive memories of a traumatic event, persistent avoidance of stimuli associated with a traumatic event, and persistent symptoms of arousal.

By the time of the publication of the DSM-5 in 2013, PTSD had been removed from the spectrum of anxiety disorders entirely and placed as the cornerstone of a new spectrum entitled "trauma and stress-related disorders," a development that the trauma subcommittee attributed to an internal disagreement about whether the features of PTSD are more organically related to features found among anxiety disorders or dissociative disorders, or whether PTSD indeed demands unique categorization. Occupying this fresh and prominent place in the new manual, the three traditional cardinal symptoms of PTSD were expanded to four, to include "avoidance and numbing." "Avoidance and numbing," as the fourth symptom, was divided into two potential presentations: (a) patterned avoidance, and (b) persistent negative alterations in cognition or mood. It is with regard to these potential presentations, newly minted in the DSM-5, that another of the five features from Chapter Two may find some connection to the psychiatric paradigm. The genesis of this fourth symptom can be found in the 1994 publication of the DSM-IV, in which there had been general discussion of the possibility of "feeling detached or estranged from others" after trauma, and of "avoidance of people that arouse recollections of trauma". The DSM-5 codified this possibility as a common feature of PTSD, framed in cognitive and meaning-attributive language as a tendency towards "negative alterations in cognition and mood associated with the traumatic event...[including] persistent and exaggerated negative 
beliefs or expectations about oneself, others, or the world, e.g. 'no one can be trusted"' [22].

Experiences of mistrust after exposure to a devastating event are usually specific - at least initially - focusing either on (a) particular people or groups of people that were also involved in the devastating event, (b) particular people or groups of people considered by the survivor to be responsible in some way for the event, or (c) particular people or groups of people whose response to the event, or whose actions in the aftermath of the event, enhanced a survivor's distress in some way. It is important to note here that the literature does acknowledge that such tendencies toward mistrust can be quite normal or ordinary, especially in the initial weeks and months after exposure to a devastating event - and in some cases it even warns caregivers to seriously consider whether assigning pathological status to specific tendencies of eventrelated mistrust in the short-term is either necessary or helpful.? Some forms of mistrust, however, especially when they persist and seem to expand to new persons and groups of persons with time, are more troubling and will likely require caring intervention. Walter McDermott, a combat veteran and clinical psychologist who has treated combat-related PTSD for more than 30 years, remembers the condition of a client who "at first learned to mistrust Vietnamese civilians, because he could not distinguish them from the enemy," but then experienced his "suspicious mistrust spreading like cancer, growing to include his leaders...other soldiers in his unit...most American politicians... and [upon his return] his wife, children, coworkers, and friends" [23]. Indeed, this progressive expansion of mistrust, beginning with one or two particularly-known perpetrators of betrayal but spreading widely and unpredictably outward across all social dimensions of a veteran's experience, seems to bear connection with the feature described above as "extreme, socially-debilitating mistrust."

Whether such extreme forms of mistrust could be summarily reduced to a "negative alteration in cognition" manifested by the "exaggeration of expectations about the world," as the language of the DSM-5 suggests, is debatable. For instance, if a warrior experiences a betrayal in combat by his leaders or teammates - which seems within the literature to be one of the most common contributing events to the eventual development of a radical form of mistrust - could this betrayal possibly occur only within the cognitive dimension of human experience, resulting in a painful form of disorientation that contains only cognitive features? It is doubtful that many caregivers would express such a belief, and, within the inter-disciplinary literature on veteran care, just as much attention is given to alteration in affectation and alteration in social enfranchisement when it comes to identifying the nature of extreme forms of veteran mistrust. For that matter, serious attention is given to meaning-attribution in the literature.

Simply by listing this tendency of extreme mistrust within the newest symptom cluster of PTSD in the DSM-5, the psychiatric paradigm demonstrates an awareness of the existence of such a phenomenon, and also demonstrates an interest in its significance to veteran care. In so doing, the psychiatric paradigm strengthens the onus for the development of more understanding of the phenomenon in years to come. The ICD-10, it should be noted, does not have any diagnostic criterion for post-traumatic stress disorder that accounts for debilitating mistrust such as has been described in accounts of the veterans in Chapter Two, with the possible exception of its references

${ }^{7}$ For instance, Bessel van der Kolk's extremely popular 2015 work The body keeps the score: brain, mind, and body in the healing of trauma warns caregivers of the weight of diagnosis in such cases, since their decisions on whether to assign a diagnosis (and which) will powerfully shape both their treatment decisions and their clients' interpretations of their own situations. to hyper-vigilance, if the meaning of that term were to be expanded to include social vigilance. However, in listing the tendency towards extreme mistrust in purely cognitive terms - something its own adherents cannot bring themselves to mimic in their publications the DSM-5 continues to provide hints about its own epistemological limitations.

Lastly, upon just a cursory examination of the potential to find an approach to self-horror at the perceived absorption of evil in combat in the DSM, it unlikely that any can be found outside of appealing to the category of "Delusional Disorder." Speaking about such things in the presence of a clinician - and especially a beginning clinician, a particularly unimaginative clinician, or a harried and overworked clinician - places a veteran in considerable danger of experiencing Jonathan Shay's most tragic prediction: the reception of a dual-diagnosis of PTSD and paranoid schizophrenia, even though a veteran may seem to have few other clear symptoms of schizophrenia [1].

\section{Episodes of lustful, exhilarating rage}

The condition described above as "episodes of lustful, exhilarating rage which resist prediction, in-state constraint, and external intervention" is one of the most spectacular features of moral injury. Although veterans experiencing such elated and strongly compulsive states sometimes claim to be able to reflect on their rage in hindsight, and to see the consequences of their actions, and even wish to change, they feel powerless to restrain themselves once the state is activated. They also feel hopeless that they can learn to sense the approach of such states, to begin got prepare to avoid them. This phenomenon is most likely to occur when (a) a veteran is engaged in a heated action where an extreme danger to his or life or a close friend's life is present, (b) when repelling that danger requires extreme physical force and intense mental focus, and (c) when the veteran is unwilling or unable to remain watchful that his or her actions in the situation remain restrained on humane grounds. If all three of these conditions are met, violent action may take an exhilarating form, and may easily violate beliefs about right action in war. From the moral injury viewpoint, once this occurs a single time, it is more likely that it will occur the next time a similar threat is presented. Eventually, if the threats in one's environment remain steady, a nearly automatic pattern can form, with disorienting consequences. Such consequences may include a veteran's engagement in violent and inhumane actions towards lesser threats, and eventually towards anything which disrupts access to his or her most powerful desires in any given moment - sometimes without reflection or moral hesitation. It may also include a turning inward, in which a veteran may experience violent episodes of self-hatred, with powerful impulses toward self-harm and suicide. Once again, I believe that this description is also consistent with a number of vignettes included in other works related to moral injury in the existing literature $[1,15,16,19,23,24$,

In the newly published PTSD symptom cluster labeled "marked alterations in arousal and reactivity associated with the traumatic event(s), beginning or worsening after the traumatic event(s) occurred," the DSM-5 addresses the tendency among returning veterans to experience "irritable behavior and angry outbursts with little or no provocation, typically expressed as verbal or physical aggression toward people or objects," and "reckless or self-destructive behavior" [25]. The American psychiatric community has long made a connection between the hyper-arousal commonly experienced within PTSD and a tendency towards extreme irritability featuring angry and potentially violent outbursts. This has been emphasized within the diagnostic criteria for PTSD, in fact, since 1980, and it is very likely that this 
emphasis provides a valid account for the unusual feature described in Chapter Two as "uncontrollable, lustful rage." Steven Taylor helpfully describes the potential for the PTSD criterion of "irritability with angry outbursts" to be extreme and completely unpredictable in some cases, even developing into episodes during which veterans can be so dazzled by murderous impulses that "things [can seem] unreal" and that they can be "in a daze," barely aware of their immediate environment [26]. This potential is certainly similar to the description I gave, above, with regard to the feature I have called "episodes of lustful, exhilarating rage which resist prediction, in-state constraint, and external intervention."

Nevertheless, some voices in the literature have questioned whether trauma-related hyper-arousal alone is capable of functioning as the primary underlying cause of all rage-filled experiences among returning veterans. ${ }^{8}$ Jonathan Shay is perhaps the most influential of these voices $[27,28]$. According to Shay, when veterans develop certain patterns of episodic violence after homecoming, such patterns can be so unalterably destructive to their psychological character that imprecise terms such as "anger" or "irritability" - even when linked with persistent hyperarousal - cannot begin to adequately describe their experiences. In Shay's opinion, certain passages within ancient Greek and Norse epic poetry come closer than the psychiatric paradigm to accounting for the multi-dimensional nature of rage in combat and its after-effects, because (although such works lack a sophisticated medical/biological anthropology) they contain a sophisticated philosophical anthropology which better represents the intangible impact of the phenomenon upon the psyche of the warrior. ${ }^{9}$ For instance, according to Shay, epic poetry contains insight into the narrative and meaning-attributive dimensions of rage in and after combat, from the warrior's own context, whereas the DSM criteria seems to take authoritative responsibility for meaningmaking according to its own presuppositions and outside of the warrior's context, claiming "ethical and culturally neutral knowledge" of what constitutes normal and abnormal patterns of anger and irritability, but failing to "clearly classify [this] abnormality in a way that fully connects with the actual experience of trauma survivors".

Shay also notes that, in his own work, abnormal veteran experiences of rage - from a psychiatric viewpoint - often resist clear containment within the psychiatric categories, because they contain features other than anger and irritability which are not covered in the symptomology of PTSD, such as strong concurrent delusions of invincibility, sexual arousal, and the potential for complete loss of short-term memory. He notes that these aspects, when presented in psychiatric interviews, often lead to concurrent clinical diagnoses, and he cites a number of instances where his veteran clients were dual-diagnosed with PTSD and schizophrenia, or PTSD and a personality disorder, muddying the potential for a nuanced understanding of the warrior's condition [1]. Edward Tick also questions the philosophical capacity of the psychiatric paradigm to account for the experience of violent rage in and after combat from which, as he describes, "there may be no return" after a war [19]. In his experience, "conventional models of medical and psychological functioning and therapeutics are not adequate to explain or treat such wounds," and he expresses a disappointment in the psychiatric community for claiming an authoritative account of this phenomenon when "the veterans and their afflictions try to tell us"

${ }^{8}$ For a sample of these writers, see the following two prominent sources: [27,28] (1) Nash WP, Carper TL, \& Mills, MA (2008), Psychometric evaluation of the moral injury events scale, Military Medicine, 17; Drescher, KD, Foy DW, Kelly C, Leshner A, Schulz K, Litz $\mathrm{B}$, An exploration of the viability and usefulness of the construct of moral injury in war veterans Traumatology 2011(17).

${ }^{9}$ This is a central tenant in his landmark work (1994) Achilles in Vietnam: combat trauma and the undoing of character. that something more than the psychiatric criteria are needed for a full understanding [29]. He also notes that the actual accounts of warriors, when it comes to having experienced something like a "berserk state" in combat, are so full of language about the supernatural world that listeners are compelled to accept that they are either having real religious experiences at the height of their disorientation, or else do not have any other language to approximate their condition. Needless to say, the psychiatric paradigm is not constructed in such a way that is capable of seriously encountering religious phenomena.

The psychiatric paradigm clearly attempts to account for - and to provide an avenue of approach towards - the tendency for some veterans to experience persistent, unpredictable, episodic rages and violent outbursts upon homecoming. This avenue of approach is one which focuses on the physiological and affective dimensions of the phenomenon, and some caregivers may be satisfied, according to the scope of their work and the boundaries of their own epistemological presuppositions, to remain within these dimensions when working with their veteran clients. However, there are some dangers inherent in doing so, especially when a veteran's patterns of irritability and rage begin to develop extremely unusual characteristics, which may become increasingly pervasive throughout the entire personality - as both Shay and Tick observed in their clients. Certainly such clients experience "alterations in arousal and reactivity," and these terms are sufficiently broad to warrant some creativity in discerning their limits, but longterm changes in personality and character are hardly contained by such terms in any context. In such cases, if caregivers reach for criteria outside of PTSD - that is, criteria organic to disorders within the psychiatric paradigm that are completely distinct from PTSD - in order to fully account for all of the features of their client's episodic rage, they risk saddling their clients with additional diagnoses, none of which clearly describe the uniqueness, complexity, and totality of the phenomenon. Needless to say, a client with two, three, or four diagnoses requires the development of a complicated and layered treatment plan - which may indicate an equally complicated and layered approach to psychopharmacological support. In such cases it seems painfully unclear whether or not a veteran's caregivers will do better than harm.

\section{Is the psychiatric paradigm sufficient?}

Strictly working within the diagnostic criteria found in the DSM5 and ICD-10, the psychiatric paradigm has significant difficulty in accounting for the complexity, uniqueness, and totality of the three unusual features under consideration in this article. Working within the widest epistemological limits of the psychiatric manuals, these three features - while to some degree approachable - demand the use of criteria from three distinct psychiatric disorders. Two of the features have significant connection to PTSD criteria, but one of these two also could be seen to have a tenuous connection with a criterion listed under a disorder in the Schizophrenia Spectrum. The third feature is related to criteria associated with disorders in the Dissociative Spectrum rather than with PTSD.

It seems the greatest complications of viewing features of moral injury through the lens of the DSM may be related to the fact that these features simply do not meet enough criteria in any one spectrum to qualify for any existing disorder - at least if the practice of diagnosis is approached in a conscientious and disciplined manner. There is no need to discuss here the dangers associated with using criteria from three distinct diagnostic spectra to try to account within the psychiatric paradigm for something that is so clearly resistant to any one psychiatric category. However, the possibility should be noted that the limitations of 
the structure of the current DSM and ICD manuals and their categories are far from the psychiatric paradigm's most salient barriers to fully accounting for the experiences of veterans struggling after moral injury. Far more salient might be the epistemological limitations of psychiatry in general.

In my experience, there are three distinct dangers present for both caregivers and clients when unusual forms of distress seem to successfully challenge the epistemological limits of the psychiatric paradigm: (a) when caregivers sense that the manuals are inadequate, they sometimes still feel pressure to use the psychiatric paradigm as a sole or primary diagnostic tool, and in this case they tend to expand the categories past their intended limits, resulting in an undermining of what real efficacy the manuals possess, (b) when language with seemingly paranormal or supernatural overtones are presented, some caregivers tend to place them into some of the most extreme psychiatric spectra, such as the schizophrenic spectrum, in order to attempt to account for them psychiatrically, and (c) when features seem to contain primary elements shaped by personal belief, or when features of distress include primary concerns about cosmic coherence or meta-level meaning-attribution, caregivers often fail to address these concerns as primary.

Given these significant dangers, it is not surprising that, as the opening of this article notices, there exists a growing body of voices in the literature questioning the efficacy of the psychiatric paradigm when used as a sole or in some cases even a primary resource for approaching unusual forms of veteran distress such as those often associated with moral injury. It should be equally unsurprising that there are a growing number of caregivers in the field who, for the reasons listed above, feel compelled to broaden their perspectives in understanding and helping veterans with extreme and unusual features of distress and disorientation upon homecoming [22,25,28,29,30-34]. Among those who have become uncomfortable using the psychiatric paradigm alone, some have chosen to supplement psychiatric criteria with wider philosophical approaches to their clients' experiences. Renos Papadapoulos uses ancient Greek texts on homecoming and Jungian constructions about the archetype of the hero to map elements of consciousness in the experience of war, dislocation, and relocation. Shay uses ancient epic war poetry as a way of expanding his understanding of the impact of war on the psyche and on social reintegration (1994). Edward Tick uses a combination of epic poetry, war fiction, and existential philosophy to understand his veteran clients [19]. Stephen Muse uses both clinical constructs from psychiatry and ancient theological constructs from Eastern Orthodox Christianity to accomplish the same goal. In expanding their conceptual base when approaching disoriented veterans, these caregivers are not dismissing the psychiatric paradigm altogether [31-33]. Through the psychiatric paradigm's reliance on scientific and medical anthropology, and through its development of extensive research into evidence-based behavioral health treatment, it captures many of the physiological and cognitive features of veteran disorientation quite adequately, and offers undeniably helpful insights and guidelines in the care of veterans. However, each of these caregivers listed above has joined the growing body of professional voices that are declaring the psychiatric paradigm to be lacking in its ability to account for the totality, uniqueness, and complexity of the disorientation of the entire population of returning veterans - and are therefore willing to expand their epistemological base through the addition of supplementary lenses.

In the past twenty-five years, perhaps no prominent psychiatrist has been more effectively outspoken on the philosophical self-limitations of psychiatry and the effects of those limitations on care to disoriented combat veterans than Jonathan Shay. Shay's development of the "moral injury" paradigm as an extension of psychiatry which offers a complementary philosophical path to understanding the suffering of veterans with unusual and morally-significant features has gained such traction in the field that it has become an essential feature of programdevelopment at Department of Veterans Affairs (VA) psychiatric hospitals and outpatient clinics throughout the United States. His moral injury paradigm, which is still underdeveloped in its nosology, does allow caregivers who have explored the philosophical limitations of psychiatric care to identify what dimension(s) of the experiences of their veteran clients is inadequately understood, and to consider what avenues of understanding are available for mobilization from other collections of knowledge and experience in the world into the gap. It is my hope that more theorists and caregivers interested in the moral injury paradigm will add their list of essential features to mine, and will undertake a similar critique of capability of the psychiatric paradigm to adequately approach and address those features. It is only when a strong distinction emerges between the features of moral injury and the latest understanding of PTSD symptoms that moral injury will be able to stand on its own as a separate syndrome, and receive the wide attention that is deserves.

\section{Acknowledgements}

None; N/A

\section{Funding information}

None; N/A

\section{Competing interests}

None to report; N/A

\section{References}

1. Shay J (1994) Achilles in Vietnam: combat trauma and the undoing of character Maxwell Macmillan International, New York, NY.

2. Alexander (2018) Gregory is my friend: on relating to veterans who claim to have absorbed evil in combat. Moral injury: a reader Meagher R (Ed) Eugene, OR: Cascade Books.

3. Hautzinger S, Scandlyn J (2013) Beyond post-traumatic stress disorder: homefront struggles with the war on terror. Walnut Creek, CA: Left Coast Press.

4. Cantrell BC, Dean C (2005) Down range: to Iraq and back. Seattle. Wordsmith Publishing, New York, NY.

5. van der Kolk BA (2015) The body keeps the score: brain, mind, and body in the healing of trauma. Penguin Books, New York.

6. Neziroglu F, Donnelly K (2010) Depersonalization disorder: a mindfulness and acceptance guide to conquering feelings of numbness and unreality. New Harbinger Publications, Oakland, CA.

7. Steinberg M (2004) Systematic assessment of post-traumatic dissociation. Assessing psychological trauma and PTSD. the Guilford Press, New York.

8. American Psychiatric Association (2013) Diagnostic and statistical manual of mental disorders. (5th Edn) American Psychiatric Association, Arlington, VA.

9. Ulman RB, Brothers D (1993) The shattered self: a psychoanalytic study of trauma Routledge Press, London.

10. Dorahy MJ, ven der Hart O (2007) Relationship between trauma and dissociation: a historical analysis. Traumatic dissociation: neurobiology and treatment. American Psychiatric Publishing, Arlington VA.

11. Wilcox V (2000) Burnout in military personnel. Military psychiatry. Department of Defense, Washington, DC.

12. Diehl TS, Goldberg K (2003) Psychiatric nursing. Philadelphia: Lippencott, Williams, \& Wilkins.

13. World Health Organization (1993) The ICD-10 classification of mental and behavioral disorders. International statistical classification of diseases and related health problems. (10th Edn) World Health Organization, Geneva. 
14. Segrest T (2008) Reflections of PTSD: with my perfect flaws. Bloomington, IN: Indiana University Press.

15. Sherman N (2011) The untold war: inside the hearts, minds, and souls of our soldiers. WW Norton Publishers, New York.

16. Hoop DC (2010) PTSD, the struggle from within: from Saigon to Baghdad. Lulu Press, Raleigh, NC.

17. McLay R (2012) At war with PTSD. Johns Hopkins University Press, Baltimore, MD.

18. Wizelman L (2011) When the war never ends: the voices of military members with PTSD and their families. Rowman and Littlefield, Lanham, MD.

19. Tick E (2005) War and the soul: healing our nation's veterans from post-traumatic stress disorder. Quest Books, Wheaton, IL.

20. DSM (1980) Diagnostic and statistical manual of mental disorders. (3rd Edn) American Psychiatric Association. Arlington, VA.

21. McCullough JP Jr, Klein DN, Borian FE, Howland RH, Riso LP, et al. (2003) Group comparisons of DSM-IV subtypes of chronic depression: validity of the distinctions, part 2. J Abnorm Psychol 112: 614-622. [Crossref]

22. Maj M (2014) DSM-5, ICD-11 and 'pathologization of normal conditions'. Aust $N Z$ Psychiatry 48: 193-194. [Crossref]

23. McDermott Walter F (2012) Understanding combat related post-traumatic stress disorder. McFarland Press, Jefferson, NC.

24. Philipps D (2010) Lethal warriors: when the new band of brothers came home: uncovering the tragic reality of PTSD. Macmillan Publishers, New York.
25. Kagan J (2012) Psychology's ghosts: the crisis in the profession and the way back. Yale University Press, New Haven, CT.

26. Taylor S (2006) Clinician's guide to PTSD: a cognitive-behavioral approach. the Guilford Group, New York.

27. Nash WP, Marino Carper TL, Mills MA, Au T, Goldsmith A, et al. (2013) Psychometric evaluation of the Moral Injury Events Scale. Mil Med 178: 646-652. [Crossref]

28. Drescher KD, Foy DW, Kelly C, Leshner A, Schulz K, et al. (2011) An exploration of the viability and usefulness of the construct of moral injury in war veterans. Traumatology 2011: 8-13.

29. Wright HW, Cummings NA (2005) Destructive trends in mental health: the wellintentioned path to harm. Routledge, London.

30. Papadopoulos RK (2007) Refugees, Trauma, and Adversity Activated Development European Journal of Psychotherapy and Counseling 9: 301-312.

31. Muse S (2013) Moral Injury. An open lecture given on May 12, 2013 at the Pentagon Memorial Auditorium. Used with permission from the author's notes.

32. Skolbekken JA (2008) Unlimited medicalization? Risk and the pathologization of normality. Health, risk and vulnerability. Routledge Press, New York.

33. Patil T, Giordano J (2010) On the ontological assumptions of the medical model of psychiatry: philosophical considerations and pragmatic tasks. Philos Ethics Humanit Med 5: 3. [Crossref]

34. Radden J (1994) Recent criticism of psychiatric nosology: a review. Philosophy, Psychiatry, \& Psychology 1: 193-200.

Copyright: (C2018 Aliyev NA. This is an open-access article distributed under the terms of the Creative Commons Attribution License, which permits unrestricted use, distribution, and reproduction in any medium, provided the original author and source are credited. 